\title{
Global exponential periodicity and stability of recurrent neural networks with multi-proportional delays
}

\begin{abstract}
In this paper, a class of recurrent neural networks with multi-proportional delays is studied. The nonlinear transformation transforms a class of recurrent neural networks with multi-proportional delays into a class of recurrent neural networks with constant delays and time-varying coefficients. By constructing Lyapunov functional and establishing the delay differential inequality, several delay-dependent and delay-independent sufficient conditions are derived to ensure global exponential periodicity and stability of the system. And several examples and their simulations are given to illustrate the effectiveness of obtained results.
\end{abstract}

Key words: Recurrent neural networks (RNNs); Proportional delay factor; Global exponential periodicity; Global exponential stability; Nonlinear transformation

\section{Introduction}

In recent years, delayed recurrent neural networks (DRNNs), such as delayed cellular neural networks, delayed Hopfield neural networks and delayed CohenGrossberg neural networks, have received great attention due to their wide applications in classification of image processing, pattern recognition, signal processing, associative memories, optimization problem, and so forth. And such applications depend on the stability of the equilibrium of the designed networks. Up to now, many results on stability of DRNNs have been developed [1-7]. At the same time, other dynamical properties of DRNNs have been widely studied, such as periodicity, synchronization and oscillation, etc. It is well known that an equilibrium point can be viewed as a special periodic solution of neural networks with arbitrary period. In this sense, the stability analysis of periodic solutions of DRNNs may be considered to be more general than that of equilibrium points. At present, a lot of periodicity results for DRNNs are obtained in [8-26].

Time delays considered for RNNs can be classified as constant delays [9$11,18,22,23,25,26]$, time-varying delays $[8,12,14,21,24]$, distributed delays [13,15- 
17,19,20]. Proportional delay is a kind of time-varying unbounded delay which is different from the above mentioned delays. Proportional delay is a kind of objective existence, for example, in Web quality of service (QoS) routing decision, the proportional delay is usually required $[27,28]$. Since a neural network usually has a spatial nature due to the presence of an amount of parallel pathways of a variety of axon sizes and lengths, it is desired to model by introducing continuously proportional delay. As an important mathematical model, the proportional delay system often rises in some fields such as physics, biology systems and control theory [29-31]. With the increase of time, the proportional delay function $(1-q) t$ is monotonically increasing, thus it may be convenient to control the network's running time according to the allowed delays. From the viewpoint of time delay, RNNs with proportional delays are different from above other DRNNs, so those obtained dynamics properties of DRNNs [8-26] cannot be directly applied to RNNs with proportional delays. It is important to ensure that the designed network is stable in the presence of proportional delay. Therefore, it has important theoretical significance to study dynamic behavior of NNs with proportional delays.

To the best of our knowledge, so far a few works have been done on the dynamical behaviors for the neural networks with proportional delays [32-38]. In [32-38], by a nonlinear transformation, a class of neural networks with proportional delays is transformed equivalently into a class of neural networks with constant delays and time-varying coefficients, then using such approaches as Lyapunov functional [19-21,23,29], algebraic inequalities [15,18,22,25,26], matrix theory [24], and so on, the their dynamics behaviors are studied. In [32], dissipativity of a class of cellular neural networks (CNNs) with proportional delays was investigated by using the inner product properties. Zhou in [3335] had discussed the global exponential stability and asymptotic stability of CNNs with proportional delays by employing matrix theory and constructing Lyapunov functional, respectively. In [36], by constructing appropriate delay differential inequalities, several new delay-independent and delay-dependent global exponential stability sufficient conditions of equilibrium point of the BAM neural networks with proportional delays were obtained. In [37], the stability criteria for high-order networks with proportional delay was studied based on matrix measure and Halanay inequality. In [38], new explicit conditions ensuring that the state trajectories of the system do not exceed a certain threshold over a pre-specified finite time interval was obtained by matrix inequalities. In this case, it is interesting and challenging to further study the periodic solutions of neural networks with proportional delays. But the dynamical behavior of periodic solutions of DRNNs with proportional delays has never been tackled.

Motivated by the discussion above, the previous criteria for checking the stability and periodicity of the addressed neural networks are somewhat conservative due to the construction of Lyapunov functionals and the technicality 
of the mathematical method used. This paper attempts to fill the gap by considering RNNs with multi-proportional delays. In this paper, criteria on the global exponential periodicity and stability of RNNs with multi-proportional delays can be derived by constructing a suitable Lyapunov functional and establishing a delay differential inequality. The delay differential inequality is established in this paper, which is similar to the Halanay inequality $[18,22,26]$ or generalized Halanay inequality [15], but it is not Halanay inequality or generalized Halanay inequality. This method might have an important role in studying the dissipativity, instability, and synchronization for complex neural networks with proportional delays.

The remainder of this paper is organized as follows. Model description and preliminaries are given in Section 2. In Section 3, several delay-dependent and delay-independent sufficient conditions are given by constructing appropriate Lyapunov functional and delay differential inequality. Numerical examples and their simulations are presented in Section 4 to illustrate the effectiveness of obtained results. Finally, conclusions are given in Section 5.

Notations: $\mathbb{R}$ denotes real number. $\mathbb{R}^{n}$ denotes $\mathrm{n}$ dimensions Euclidean space. Let $\bar{C}=\bar{C}\left([q, 1] ; \mathbb{R}^{n}\right)$ be the Banach space of all continuous function from $[q, 1]$ to $\mathbb{R}^{n}$ with the topology of uniform convergence. For any $\varphi(s) \in \bar{C}$, let $\|\varphi(s)\|=\sum_{i=1}^{n} \sup _{q \leq s \leq 1}\left|\varphi_{i}(s)\right|$. Let $C=C\left([-\tau, 0] ; \mathbb{R}^{n}\right)$ be the Banach space of all continuous function from $[-\tau, 0]$ to $\mathbb{R}^{n}$ with the topology of uniform convergence. For any $\psi \in C$, let $\|\psi(t)\|=\sum_{i=1}^{n} \sup _{-\tau \leq t \leq 0}\left|\psi_{i}(t)\right|$. Define $x_{t}=x(s+t)$, $x_{t}\left(x_{0}\right)=x\left(s+t, x_{0}\right), \quad s \in[q, 1], t \geq 1$. Denote $\left\|x_{t}\right\|=\sum_{i=1}^{n} \sup _{q \leq s \leq 1}\left|x_{i}(s+t)\right|$. Define $y_{t}=y(t+\theta), y_{t}(\varphi)=y(t+\theta, \varphi), \theta \in[-\tau, 0], \quad t \geq 0$. Denote $\left\|y_{t}\right\|=\sum_{i=1}^{n} \sup _{-\tau \leq \theta \leq 0}\left|y_{i}(t+\theta)\right|$.

\section{System Description and Preliminaries}

Consider a class of recurrent neural networks with multi-proportional delays described by

$$
\left\{\begin{aligned}
& \dot{x}_{i}(t)=-d_{i} x_{i}(t)+ \sum_{j=1}^{n}\left[a_{i j} f_{j}\left(x_{j}(t)\right)+\right. \\
&\left.b_{i j} g_{j}\left(x_{j}\left(p_{j} t\right)\right)+c_{i j} h_{j}\left(x_{j}\left(q_{j} t\right)\right)\right]+I_{i}(t), \\
& x_{i}(s)=\varphi_{i}(s), s \in[q, 1]
\end{aligned}\right.
$$


for $i=1,2, \cdots, n, t \geq 1$. In (2.1), $x_{i}(t)$ denotes the state variable of the $i$ th neuron at time $t ; d_{i}>0$ denotes the rate with which the $i$ th neuron resets its potential to the resting state when isolated from the other neuron and inputs; $a_{i j}, b_{i j}$ and $c_{i j}$ denote the connection weights between the $i$ th neuron and the $j$ th neuron at time $t, p_{j} t$ and $q_{j} t$, respectively; $p_{j}$ and $q_{j}$ are proportional delay factors and satisfy $0<p_{j}, q_{j} \leq 1, p_{j} t=t-\left(1-p_{j}\right) t, q_{j} t=t-\left(1-q_{j}\right) t$, in which $\left(1-p_{j}\right) t$ and $\left(1-q_{j}\right) t$ denote the transmission delays; $q=\min _{1 \leq j \leq n}\left\{p_{j}, q_{j}\right\} ; I_{i}(t)$ is the external input periodic function with period $\omega$, i.e. there exists a constant $\omega>0$ such that $I_{i}(t+\omega)=I_{i}(t), i=1,2, \cdots, n ; \varphi_{i}(s)$ is a initial function at $s \in[q, 1], \varphi(s)=\left(\varphi_{1}(s), \varphi_{2}(s), \cdots, \varphi_{n}(s)\right)^{T} \in C\left([q, 1], \mathbb{R}^{n}\right) . f_{i}(\cdot), g_{i}(\cdot)$ and $h_{i}(\cdot): \mathbb{R} \rightarrow \mathbb{R}$ are the nonlinear activation functions, and assumed to satisfy the following requirements

$$
\left\{\begin{array}{l}
\left|f_{i}(u)-f_{i}(v)\right| \leq L_{i}|u-v| \\
\left|g_{i}(u)-g_{i}(v)\right| \leq M_{i}|u-v| \\
\left|h_{i}(u)-h_{i}(v)\right| \leq N_{i}|u-v|
\end{array}\right.
$$

for $i=1,2, \cdots, n, u, v \in \mathbb{R}$. Where $L_{i}, M_{i}, N_{i}$ are nonnegative constants.

As a special case of (2.1), the DRNNs with constant input $I_{i}$ is described by the following functional differential equations:

$$
\left\{\begin{aligned}
& \dot{x}_{i}(t)=-d_{i} x_{i}(t)+ \sum_{j=1}^{n}\left[a_{i j} f_{j}\left(x_{j}(t)\right)+\right. \\
&\left.b_{i j} g_{j}\left(x_{j}\left(p_{j} t\right)\right)+c_{i j} h_{j}\left(x_{j}\left(q_{j} t\right)\right)\right]+I_{i}, \\
& x_{i}(s)=\varphi_{i}(s), s \in[q, 1], i=1,2, \cdots, n .
\end{aligned}\right.
$$

Remark 2.1 The transmission delays are $\left(1-p_{j}\right) t \rightarrow+\infty,\left(1-q_{j}\right) t \rightarrow$ $+\infty$, as $t \rightarrow+\infty, j=1,2, \cdots, n$ in (2.1) and (2.3), so (2.1) and (2.3) are different from models in [8-26]. Those stability results in [8-26] cannot be directly applied to (2.1).

Let us consider the following nonlinear transformation defined by

$$
y_{i}(t)=x_{i}\left(\mathrm{e}^{t}\right)
$$

then it is easy to prove that systems (2.1) and (2.3) are equivalent to the following RNNs with constant delays and time-varying coefficients, respectively 
(see, [33]).

$$
\left\{\begin{array}{l}
\dot{y}_{i}(t)=\mathrm{e}^{t}\left\{-d_{i} y_{i}(t)+\sum_{j=1}^{n}\left[a_{i j} f_{j}\left(y_{j}(t)\right)+\right.\right. \\
\left.\left.b_{i j} g_{j}\left(y_{j}\left(t-\tau_{j}\right)\right)+c_{i j} h_{j}\left(y_{j}\left(t-\varsigma_{j}\right)\right)\right]+u_{i}(t)\right\}, \\
y_{i}(s)=\psi_{i}(s), s \in[-\tau, 0],
\end{array}\right.
$$

and

$$
\left\{\begin{array}{l}
\dot{y}_{i}(t)=\mathrm{e}^{t}\left\{-d_{i} y_{i}(t)+\sum_{j=1}^{n}\left[a_{i j} f_{j}\left(y_{j}(t)\right)+\right.\right. \\
\left.\left.b_{i j} g_{j}\left(y_{j}\left(t-\tau_{j}\right)\right)+c_{i j} h_{j}\left(y_{j}\left(t-\varsigma_{j}\right)\right)\right]+I_{i}\right\}, \\
y_{i}(s)=\psi_{i}(s), \quad s \in[-\tau, 0],
\end{array}\right.
$$

for $t \geq 0, i=1,2, \cdots, n$. Where $\tau_{j}=-\log p_{j} \geq 0, \varsigma_{j}=-\log q_{j} \geq 0, \tau=$ $\max _{1 \leq j \leq n}\left\{\tau_{j}, \varsigma_{j}\right\}, u_{i}(t)=I_{i}\left(\mathrm{e}^{t}\right) . \psi_{i}(s)=\varphi_{i}\left(\mathrm{e}^{s}\right) \in C([-\tau, 0], \mathbb{R}), \quad i=1,2, \cdots, n$. $\psi=\left(\psi_{1}, \psi_{2}, \cdots, \psi_{n}\right)^{T} \in C\left([-\tau, 0], \mathbb{R}^{n}\right)$.

Remark 2.2 (2.5) and (2.6) are different from models in [14,15,21,24]. The coefficients of models in [14,15,21,24] are bounded time variable functions, but the coefficients which contain $e^{t}$ in (2.5) and (2.6) are unbounded time-varying functions.

Let $x^{*}=\left(x_{1}^{*}, x_{2}^{*}, \cdots, x_{n}^{*}\right)^{T}$ be the equilibrium point of system (2.3), we denote $\left\|\varphi-x^{*}\right\|=\sum_{i=1}^{n} \sup _{q \leq s \leq 1}\left|\varphi_{i}(s)-x_{i}^{*}\right|$. Let $x(t)$ be an arbitrary solution of system $(2.3)$ for $t \geq 1$, where $x(t)=\left(x_{1}(t, \varphi), x_{2}(t, \varphi), \cdots, x_{n}(t, \varphi)\right)^{T}$.

Let $y^{*}=\left(y_{1}^{*}, y_{2}^{*}, \cdots, y_{n}^{*}\right)^{T}$ be the equilibrium point of system (2.6), we denote $\left\|\psi-y^{*}\right\|=\sum_{i=1}^{n} \sup _{-\tau \leq \theta \leq 0}\left|\psi_{i}(\theta)-y_{i}^{*}\right|$. Let $y(t)$ be an arbitrary solution of system (2.6) for $t \geq 0$, where $y(t)=\left(y_{1}(t, \psi), y_{2}(t, \psi), \cdots, y_{n}(t, \psi)\right)^{T}$.

Definition 2.3 An equilibrium point $x^{*} \in \mathbb{R}^{n}$ of system (2.3) is said to be globally exponentially stable if there exist two positive constants $\beta \geq 1$ and $\alpha>0$ such that

$$
\left\|x(t)-x^{*}\right\| \leq \beta\left\|\varphi-x^{*}\right\| t^{-\alpha}, t \geq 1,
$$

for any $x(t) \in \mathbb{R}^{n}$ with $\left\|x(t)-x^{*}\right\|=\sum_{i=1}^{n}\left|x_{i}(t)-x_{i}^{*}\right|$.

Definition 2.4 [17] System (2.1) is said to be globally exponentially periodic if there exists one $\omega$-periodic solution of system (2.1) and all other solutions of the system converge exponentially to it as $t \rightarrow \infty$. 


\section{Main Results}

Given any $\varphi(s), \varphi \overline{(s)} \in \bar{C}$, let $x(t, \varphi)=\left(x_{1}(t, \varphi), x_{2}(t, \varphi), \cdots, x_{n}(t, \varphi)\right)^{T}$ and $x\left(t, \bar{\varphi}_{s}\right)=\left(x_{1}(t, \bar{\varphi}), x_{2}(t, \bar{\varphi}), \cdots, x_{n}(t, \bar{\varphi})\right)^{T}$ be the solutions of $(2.1)$ starting from $\varphi$ and $\bar{\varphi}$, respectively.

Given any $\psi, \bar{\psi} \in C$, let $y(t, \psi)=\left(y_{1}(t, \psi), y_{2}(t, \psi), \cdots, y_{n}(t, \psi)\right)^{T}$ and $y(t, \bar{\psi})=\left(y_{1}(t, \bar{\psi}), y_{2}(t, \bar{\psi}), \cdots, y_{n}(t, \bar{\psi})\right)^{T}$ be the solutions of $(2.5)$ starting from $\psi$ and $\bar{\psi}$, respectively.

Theorem 3.1 Under condition (2.2), if there exist a positive number $\sigma>1$, such that

$$
d_{i}-\sigma-\sum_{j=1}^{n}\left(\left|a_{j i}\right| L_{i}+\left|b_{j i}\right| e^{\sigma \tau_{i}} M_{i}+\left|c_{j i}\right| e^{\sigma \varsigma_{i}} N_{i}\right) \geq 0
$$

for $i=1,2, \cdots, n$, then for every periodic input $I_{i}(t)$, system (2.1) is globally exponentially periodic. Where $\tau_{i}=-\log p_{i} \geq 0, \varsigma_{i}=-\log q_{i} \geq 0$.

Proof. By $y_{t}(\psi)=y(t+\theta, \psi)$, then $y_{t}(\psi) \in C$ for all $t \geq 0$. By using (2.2), it follows from $(2.5)$ that

$$
\begin{aligned}
& D^{+}\left|y_{i}(t, \psi)-y_{i}(t, \bar{\psi})\right| \leq \mathrm{e}^{t}\left\{-d_{i} \mid\left(y_{i}(t, \psi)-y_{i}(t, \bar{\psi}) \mid\right.\right. \\
& \quad+\sum_{j=1}^{n}\left|a_{i j}\right| L_{j}\left|y_{j}(t, \psi)-y_{j}(t, \bar{\psi})\right|+\sum_{j=1}^{n}\left|b_{i j}\right| M_{j}\left|y_{j}\left(t-\tau_{j}, \psi\right)-y_{j}\left(t-\tau_{j}, \bar{\psi}\right)\right| \\
& \left.\quad+\sum_{j=1}^{n}\left|c_{i j}\right| N_{j}\left|y_{j}\left(t-\varsigma_{j}, \psi\right)-y_{j}\left(t-\varsigma_{j}, \bar{\psi}\right)\right|\right\}
\end{aligned}
$$

for $t \geq 0, i=1,2, \cdots, n$. Where $D^{+}$denotes the upper right derivation.

Accordingly, we then consider function $Y_{i}(t)$ defined by

$$
Y_{i}(t)=\mathrm{e}^{\sigma t}\left|y_{i}(t, \psi)-y_{i}(t, \bar{\psi})\right|
$$

Substituting (3.3) into (3.2) yields

$$
\begin{aligned}
D^{+} Y_{i}(t) \leq \sigma Y_{i}(t)-d_{i} \mathrm{e}^{t} Y_{i}(t)+\mathrm{e}^{t}\left\{\sum_{j=1}^{n}\left|a_{i j}\right| L_{j} Y_{j}(t)+\right. \\
\left.\sum_{j=1}^{n}\left|b_{i j}\right| M_{j} Y_{j}\left(t-\tau_{j}\right) \mathrm{e}^{\sigma \tau_{j}}+\sum_{j=1}^{n}\left|c_{i j}\right| N_{j} Y_{j}\left(t-\varsigma_{j}\right) \mathrm{e}^{\sigma \varsigma_{j}}\right\},
\end{aligned}
$$


for $i=1,2, \cdots, n$. We then construct a Lyapunov functional of the form

$$
\begin{gathered}
V(t)=\sum_{i=1}^{n}\left\{\mathrm{e}^{-t} Y_{i}(t)+\sum_{j=1}^{n}\left(\left|b_{i j}\right| M_{j} \int_{t-\tau_{j}}^{t} \mathrm{e}^{\sigma \tau_{j}} Y_{j}(s) \mathrm{d} s+\right.\right. \\
\left.\left.\left|c_{i j}\right| N_{j} \int_{t-\varsigma_{j}}^{t} \mathrm{e}^{\sigma \varsigma_{j}} Y_{j}(s) \mathrm{d} s\right)\right\},
\end{gathered}
$$

for $t \geq 0, \sigma>1$, and calculating the rate of change $D^{+} V(t)$ of $V(t)$ along (3.4)

$$
\begin{aligned}
& D^{+} V(t) \\
& \leq \sum_{i=1}^{n}\left\{-\mathrm{e}^{-t} Y_{i}(t)+\mathrm{e}^{-t}\left[\sigma Y_{i}(t)-d_{i} \mathrm{e}^{t} Y_{i}(t)+\right.\right. \\
&\left.\left.\sum_{j=1}^{n}\left|a_{i j}\right| L_{j} \mathrm{e}^{t} Y_{j}(t)+\sum_{j=1}^{n}\left|b_{i j}\right| M_{j} \mathrm{e}^{t} Y_{j}(t) \mathrm{e}^{\sigma \tau_{j}}+\sum_{j=1}^{n}\left|c_{i j}\right| N_{j} \mathrm{e}^{t} Y_{j}(t) \mathrm{e}^{\sigma \varsigma_{j}}\right]\right\} \\
&=-\sum_{i=1}^{n}\left[(1-\sigma) \mathrm{e}^{-t}+d_{i}-\sum_{j=1}^{n}\left(\left|a_{j i}\right| L_{i}+\left|b_{j i}\right| M_{i} \mathrm{e}^{\sigma \tau_{i}}+\left|c_{j i}\right| N_{i} \mathrm{e}^{\sigma \varsigma_{i}}\right)\right] Y_{i}(t) \\
& \leq-\sum_{i=1}^{n}\left[\left(d_{i}-\sigma\right)-\sum_{j=1}^{n}\left(\left|a_{j i}\right| L_{i}+\left|b_{j i}\right| M_{i} \mathrm{e}^{\sigma \tau_{i}}+\left|c_{j i}\right| N_{i} \mathrm{e}^{\sigma \varsigma_{i}}\right)\right] Y_{i}(t) .
\end{aligned}
$$

By applying (3.1) in the above inequality, we deduce that $D^{+} V(t) \leq 0$ for $t \geq 0$ with the implication $V(t) \leq V(0)$ for $t \geq 0$. Using this and (3.5), we have

$$
\sum_{i=1}^{n} \mathrm{e}^{-t} Y_{i}(t) \leq V(t) \leq V(0)
$$

And, it follows from (3.5) that

$$
\begin{aligned}
V(0) & \leq \sum_{i=1}^{n}\left\{Y_{i}(0)+\sum_{j=1}^{n}\left(\left|b_{i j}\right| M_{j} \tau_{j} \mathrm{e}^{\sigma \tau_{j}} \sup _{-\tau_{j} \leq s \leq 0} Y_{j}(s)+\left|c_{i j}\right| N_{j} \varsigma_{j} \mathrm{e}^{\sigma \varsigma_{j}} \sup _{-\varsigma_{j} \leq s \leq 0} Y_{j}(s)\right)\right\} \\
& \leq \sum_{i=1}^{n} Y_{i}(0)+\sum_{i=1}^{n} \sum_{j=1}^{n}\left(\left|b_{j i}\right| M_{i} \tau \mathrm{e}^{\sigma \tau}+\left|c_{j i}\right| N_{i} \tau \mathrm{e}^{\sigma \tau}\right) \sup _{-\tau \leq s \leq 0} Y_{i}(s) \\
& \leq \max _{1 \leq i \leq n}\left\{\left[1+\tau \mathrm{e}^{\sigma \tau} \sum_{j=1}^{n}\left(\left|b_{j i}\right| M_{i}+\left|c_{j i}\right| N_{i}\right)\right]\right\} \sum_{i=1}^{n} \sup _{-\tau \leq s \leq 0} Y_{i}(s)
\end{aligned}
$$

where $\beta=\max _{1 \leq i \leq n}\left\{1+\tau \mathrm{e}^{\sigma \tau} \sum_{j=1}^{n}\left(\left|b_{j i}\right| M_{i}+\left|c_{j i}\right| N_{i}\right)\right\} \geq 1$. Hence, by (3.3) and (3.6), we obtain

$$
\sum_{i=1}^{n}\left|y_{i}(t, \psi)-y_{i}(t, \bar{\psi})\right| \leq \beta \mathrm{e}^{-\alpha t} \sum_{i=1}^{n} \sup _{-\tau \leq s \leq 0}\left|y_{i}(t, \psi)-y_{i}(t, \bar{\psi})\right|,
$$


for $t \geq 0$, where $\alpha=\sigma-1>0$.

Let $\psi(t)=\varphi\left(\mathrm{e}^{t}\right), \bar{\psi}(t)=\bar{\varphi}\left(\mathrm{e}^{t}\right)$, it follows from (2.4) and (3.7) that

$$
\begin{aligned}
& \sum_{i=1}^{n}\left|x_{i}\left(\mathrm{e}^{t}, \varphi\left(\mathrm{e}^{t}\right)\right)-x_{i}\left(\mathrm{e}^{t}, \bar{\varphi}\left(\mathrm{e}^{t}\right)\right)\right| \\
& \quad \leq \beta \mathrm{e}^{-\alpha t} \sum_{i=1}^{n} \sup _{-\tau \leq s \leq 0}\left|x_{i}\left(\mathrm{e}^{s}, \varphi\left(\mathrm{e}^{s}\right)\right)-x_{i}\left(\mathrm{e}^{s}, \bar{\varphi}\left(\mathrm{e}^{s}\right)\right)\right| .
\end{aligned}
$$

Let $\mathrm{e}^{t}=\eta$, then $\eta \geq 1$ and $t=\log \eta \geq 0 ;$ Let $\mathrm{e}^{s}=\xi$, then $s=\log \xi \in[-\tau, 0]$ and $\xi \in[q, 1]$. Thus, by (3.8), we get

$$
\sum_{i=1}^{n}\left|x_{i}(\eta, \varphi(\eta))-x_{i}(\eta, \bar{\varphi}(\eta))\right| \leq \beta \mathrm{e}^{-\alpha \log \eta} \sum_{i=1}^{n} \sup _{q \leq \xi \leq 1}\left|\varphi_{i}(\xi)-\bar{\varphi}_{i}(\xi)\right|, \quad \eta \geq 1
$$

Taking $\eta=t$, we obtain that

$$
\|x(t, \varphi)-x(t, \bar{\varphi})\| \leq \beta\|\varphi-\bar{\varphi}\| \mathrm{e}^{-\alpha \log t}=\beta\|\varphi-\bar{\psi}\| t^{-\alpha}, t \geq 1
$$

where $\|\varphi-\bar{\varphi}\|=\sum_{i=1}^{n} \sup _{q \leq \xi \leq 1}\left|\varphi_{i}-\bar{\varphi}_{i}\right|$. It follows from (3.10) that

$$
\left\|x_{t}(\varphi)-x_{t}(\bar{\varphi})\right\| \leq \beta\|\varphi-\bar{\varphi}\|(q t)^{-\alpha}, t \geq 1 \text {. }
$$

We can choose a positive integer $m$ such that

$$
\beta(q m \omega)^{-\alpha} \leq \frac{1}{4}
$$

Now, define a Poincaré mapping $H: \bar{C} \rightarrow \bar{C}$ by $H \varphi=x_{\omega}(\varphi)$. Then we can derive from (3.11) and (3.12) that

$$
\left\|H^{m} \varphi-H^{m} \bar{\varphi}\right\| \leq \frac{1}{4}\|\varphi-\bar{\varphi}\| .
$$

where $H^{m} \varphi=x_{m \omega}(\varphi)$. This implies that $H^{m}$ is a contraction mapping. Therefore, there exists a unique fixed point $\varphi^{*} \in \bar{C}$ such that $H^{m} \varphi^{*}=\varphi^{*}$. Note that

$$
H^{m}\left(H \varphi^{*}\right)=H\left(H^{m} \varphi^{*}\right)=H \varphi^{*}
$$

This shows that $H \varphi^{*} \in \bar{C}$ is also a fixed point of $H^{m}$, hence, $H \varphi^{*}=\varphi^{*}$, that is, $x_{\omega}\left(\varphi^{*}\right)=\varphi^{*}$. 
Let $x\left(t, \varphi^{*}\right)$ be the solution of $(2.1)$ through $\left(0, \varphi^{*}\right)$. By using $I(t+\omega)=I(t)$ for $t \geq 1, x\left(t+\omega, \varphi^{*}\right)$ is also a solution of $(2.1)$, and note that

$$
x_{t+\omega}\left(\varphi^{*}\right)=x_{t}\left(x_{\omega}\left(\varphi^{*}\right)\right)=x_{t}\left(\varphi^{*}\right),
$$

for $t \geq 1$, then $x\left(t+\omega, \varphi^{*}\right)=x\left(t, \varphi^{*}\right)$ for $t \geq 1$.

This shows that $x\left(t, \varphi^{*}\right)$ is a periodic solution of (2.1) with period $\omega$. From (3.11), it is easy to see that all other solution of (2.1) converge to this periodic solution exponentially as $t \rightarrow \infty$.

A sufficient conditions of delay-dependent global exponentially periodic for (2.1) is obtained in Theorem 3.1. In the following, we give a sufficient condition of delay-independent global exponentially periodic for (2.1).

Theorem 3.2 Under condition (2.2), if

$$
d_{i}-\sum_{j=1}^{n}\left(\left|a_{i j}\right| L_{j}+\left|b_{i j}\right| M_{j}+\left|c_{i j}\right| N_{j}\right)>0, i=1,2, \cdots, n
$$

hold, then for every periodic input $I(t)$, the system (2.1) is globally exponentially periodic.

Proof. Let us consider function $P_{i}\left(v_{i}\right)$ defined by

$$
P_{i}\left(v_{i}\right)=d_{i}-v_{i}-\sum_{j=1}^{n}\left(\left|a_{i j}\right| L_{j}+\left|b_{i j}\right| M_{j} \mathrm{e}^{v_{i} \tau_{j}}+\left|c_{i j}\right| N_{j} \mathrm{e}^{v_{i} \varsigma_{j}}\right)
$$

for $v_{i} \in[0, \infty), i=1,2, \cdots, n$. We notice from (3.14) that

$$
d_{i}-\sum_{j=1}^{n}\left(\left|a_{i j}\right| L_{j}+\left|b_{i j}\right| M_{j}+\left|c_{i j}\right| N_{j}\right) \geq \delta, i=1,2, \cdots, n,
$$

where $\delta=\min _{1 \leq i \leq n}\left\{d_{i}-\sum_{j=1}^{n}\left(\left|a_{i j}\right| L_{j}+\left|b_{i j}\right| M_{j}+\left|c_{i j}\right| N_{j}\right)\right\}>0$. It follows from (3.15) that $P_{i}(0) \geq \delta$ for $i=1,2, \cdots, n$. We observe that $P_{i}\left(v_{i}\right)$ is continuous for $v_{i} \in[0, \infty)$, and $P_{i}\left(v_{i}\right) \rightarrow-\infty$ as $v_{i} \rightarrow \infty$. Thus there exist constants $\tilde{v}_{i} \in(0, \infty), i=1,2, \cdots, n$, such that

$$
P_{i}\left(\tilde{v}_{i}\right)=d_{i}-\tilde{v}_{i}-\sum_{j=1}^{n}\left(\left|a_{i j}\right| L_{j}+\left|b_{i j}\right| M_{j} \mathrm{e}^{\tilde{v}_{i} \tau_{j}}+\left|c_{i j}\right| N_{j} \mathrm{e}^{\tilde{v}_{i} \varsigma_{j}}\right)=0 .
$$

Therefore, there must exist a positive constant $\eta \in\left(0, \min \left\{\tilde{v}_{i}\right\}\right)$, such that

$$
P_{i}(\eta)=d_{i}-\eta-\sum_{j=1}^{n}\left(\left|a_{i j}\right| L_{j}+\left|b_{i j}\right| M_{j} \mathrm{e}^{\eta \tau_{j}}+\left|c_{i j}\right| N_{j} \mathrm{e}^{\eta \varsigma_{j}}\right)>0
$$


for $i=1,2, \cdots, n$. Accordingly, we define the function $Z(\cdot)$ as follows

$$
Z_{i}(t)=\mathrm{e}^{\eta t}\left|y_{i}(t, \psi)-y_{i}(t, \bar{\psi})\right|, t \in[-\tau,+\infty) .
$$

We then use (3.2) and (3.19) to derive the following inequality for $t>0$ given by

$$
\begin{aligned}
& D^{+} Z_{i}(t) \leq \eta Z_{i}(t)-d_{i} \mathrm{e}^{t} Z_{i}(t)+\mathrm{e}^{t} \sum_{j=1}^{n}\left\{\left|a_{i j}\right| L_{j} Z_{j}(t)+\right. \\
&\left.\left|b_{i j}\right| M_{j} Z_{j}\left(t-\tau_{j}\right) \mathrm{e}^{\eta \tau_{j}}+\left|c_{i j}\right| N_{j} Z_{j}\left(t-\varsigma_{j}\right) \mathrm{e}^{\eta \varsigma_{j}}\right\} \\
& \leq-\left(d_{i}-\eta\right) \mathrm{e}^{t} Z_{i}(t)+\mathrm{e}^{t} \sum_{j=1}^{n}\left(\left|a_{i j}\right| L_{j}+\right. \\
&\left.\left|b_{i j}\right| M_{j} \mathrm{e}^{\eta \tau_{j}}+\left|c_{i j}\right| N_{j} \mathrm{e}^{\eta \varsigma_{j}}\right) \sup _{-\tau \leq s \leq t} Z_{j}(s) .
\end{aligned}
$$

Let

$$
T=\max _{1 \leq i \leq n}\left\{\sup _{-\tau \leq s \leq 0}\left|y_{i}(s, \psi)-y_{i}(s, \bar{\psi})\right|\right\}
$$

where $T>0$.

We notice from (3.21) and (3.19) that $Z_{i}(t) \leq T$ for $i=1,2, \cdots, n, t \in$ $[-\tau, 0]$, where $T>0$ is given by $(3.21)$. We claim

$$
Z_{i}(t) \leq T, i=1,2, \cdots, n, t \in[0,+\infty)
$$

Here we first prove that for any $d>1$, there are

$$
Z_{i}(t)<d T, i=1,2, \cdots, n, t \in[0,+\infty) .
$$

Suppose that this claim (3.23) does not hold in this sense that there is one component among $Z_{i}(\cdot)$ (say $Z_{k}(\cdot)$ ) and a first time $t_{1}>0$ such that

$$
Z_{k}(t)<d T, t \in\left[-\tau, t_{1}\right), Z_{k}\left(t_{1}\right)=d T, D^{+} Z_{k}\left(t_{1}\right) \geq 0 .
$$

and

$$
Z_{i}(t) \leq d T, i \neq k, t \in\left[-\tau, t_{1}\right]
$$

On the other hand, substituting (3.24) and (3.25) into (3.20) yields

$$
0 \leq D^{+} Z_{k}\left(t_{1}\right) \leq-\left(d_{k}-\eta-\sum_{j=1}^{n}\left(\left|a_{k j}\right| L_{j}+\left|b_{k j}\right| M_{j} \mathrm{e}^{\eta \tau_{j}}+\left|c_{k j}\right| N_{j} \mathrm{e}^{\eta \varsigma_{j}}\right) \mathrm{e}^{t_{1}} d T\right.
$$


and by applying (3.18) to the above inequality, we lead to $0 \leq D^{+} Z_{k}\left(t_{1}\right)<0$ which means a contradiction. Thus, for $t \geq 0, Z_{i}(t)<d T$. As $d \rightarrow 1$, we obtain that the claim (3.22) must hold. It follows (3.19) and (3.22) that

$$
\left|y_{i}(t, \psi)-y_{i}(t, \bar{\psi})\right| \leq T \mathrm{e}^{-\eta t}, i=1,2, \cdots, n, t \geq 0
$$

Let

$$
\lambda=\frac{\max _{1 \leq i \leq n}\left\{\sup _{-\tau \leq s \leq 0}\left|y_{i}(s, \psi)-y_{i}(s, \bar{\psi})\right|\right\}}{\sup _{-\tau \leq s \leq 0}\left|y_{i}(s, \psi)-y_{i}(s, \bar{\psi})\right|},
$$

then $\lambda \geq 1$. And according to (3.21) and (3.28) yields

$$
T=\lambda \sup _{-\tau \leq s \leq 0}\left|y_{i}(s, \psi)-y_{i}(s, \bar{\psi})\right|
$$

Substituting (3.29) and (3.19) into (3.22) yields

$$
\|y(t, \psi)-y(t, \bar{\psi})\| \leq \lambda\|\psi-\bar{\psi}\| \mathrm{e}^{-\eta t}, t \geq 0 .
$$

It follows from (3.30) that

$$
\left\|y_{t}(\psi)-y_{t}(\bar{\psi})\right\| \leq \lambda\|\psi-\bar{\psi}\| \mathrm{e}^{-\eta(t-\tau)} .
$$

Then, by $y_{i}(t)=x_{i}\left(\mathrm{e}^{t}\right)$, we have

$$
\left\|x_{t}(\varphi)-x_{t}(\bar{\varphi})\right\| \leq \lambda\|\varphi-\bar{\varphi}\|(q t)^{-\alpha}, t \geq 1
$$

By the same arguments as in Theorem 3.1, we obtain that $x\left(t, \varphi^{*}\right)$ is a periodic solution of (2.1) with period $\omega$. From (3.32), it is easy to see that all other solutions of (2.1) converge to this periodic solution exponentially as $t \rightarrow+\infty$.

In addition, in view of the proof of the Theorem 3.2, the following result is obtained.

Theorem 3.3 Under condition (2.2), if there exist a positive number $\eta>0$, such that

$$
d_{i}-\eta-\sum_{j=1}^{n}\left(\left|a_{i j}\right| L_{j}+\left|b_{i j}\right| e^{\eta \tau_{j}} M_{j}+\left|c_{i j}\right| e^{\eta \varsigma_{j}} N_{j}\right)>0
$$

for $i=1,2, \cdots, n$, then for every periodic input $I(t)$, system (2.1) is globally exponentially periodic. Where $\tau_{i}=-\log p_{i} \geq 0, \varsigma_{i}=-\log q_{i} \geq 0$.

Further, by Theorems 3.1, 3.2 and 3.3, we obtain the following Corollaries. 
Corollary 3.4 Under condition (2.2), if there exist a positive number $\sigma>1$, for $i=1,2, \cdots, n$ such that

$$
d_{i}-\sigma-\sum_{j=1}^{n}\left(\left|a_{j i}\right| L_{i}+\left|b_{j i}\right| M_{i} e^{-\sigma \log p_{i}}+\left|c_{j i}\right| N_{i} e^{-\sigma \log q_{i}}\right)>0
$$

hold, then the equilibrium point of system (2.3) is globally exponentially stable.

Corollary 3.5 Under condition (2.2), if

$$
d_{i}-\sum_{j=1}^{n}\left(\left|a_{i j}\right| L_{j}+\left|b_{i j}\right| M_{j}+\left|c_{i j}\right| N_{j}\right)>0, i=1,2, \cdots, n
$$

hold, then the equilibrium point of system (2.3) is globally exponentially stable.

Corollary 3.6 Under condition (2.2), if there exist a positive number $\eta>0$, for $i=1,2, \cdots, n$ such that

$$
d_{i}-\eta-\sum_{j=1}^{n}\left(\left|a_{i j}\right| L_{j}+\left|b_{i j}\right| e^{-\eta \log p_{j}} M_{j}+\left|c_{i j}\right| e^{-\eta \log q_{j}} N_{j}\right) \geq 0, i=1,2, \cdots, n
$$

hold, then the equilibrium point of system (2.3) is globally exponentially stable.

Remark 3.7 Corollary 3.4 in the paper is the same as Corollary 3.4 in [33].

Remark 3.8 In (2.1), if $p_{j}=q_{j}=1, j=1,2, \cdots, n$, then system (2.1) becomes standard RNNs without delays. Thus, results in the paper are also applicable to standard RNNs without delays.

\section{Illustrative Examples}

In the following, we will give several examples to illustrate our results.

Example 4.1 Consider the following system

$$
\dot{x}_{i}(t)=-d_{i} x_{i}(t)+\sum_{j=1}^{2}\left[a_{i j} f_{j}\left(x_{j}(t)\right)+b_{i j} f_{j}\left(x_{j}\left(p_{j} t\right)\right)+c_{i j} f_{j}\left(x_{j}\left(q_{j} t\right)\right)\right]+I_{i}(t),
$$

for $i=1,2, t \geq 1$. Where $d_{1}=d_{2}=8, a_{11}=0.5, a_{12}=-1, a_{21}=$ $-1, a_{22}=1, b_{11}=1, b_{12}=-1, b_{21}=-1, b_{22}=1.5, c_{11}=0, c_{12}=$ $1, c_{21}=-1, c_{22}=2 . I_{1}(t)=\sin (t), I_{2}(t)=\cos (t)$. The activation functions are $f_{i}\left(x_{i}\right)=\sin \left(\frac{1}{3} x_{i}\right)+\frac{1}{3} x_{i}, i=1,2$. And $p_{j}=0.4, q_{j}=0.8, j=1,2$.

Obviously $f_{i}\left(x_{i}\right), i=1,2$ are Lipschitz continuous with Lipschitz constant $L_{i}=\frac{2}{3}, i=1,2 . \tau_{j}=-\log 0.4=0.9163, \varsigma_{j}=-\log 0.8=0.2231, j=1,2$. 
Taking $\sigma=1.2$, we have

$$
\begin{aligned}
& d_{1}-\sigma-\frac{2}{3}\left[\left(\left|a_{11}\right|+\left|a_{21}\right|\right)+\left(\left|b_{11}\right|+\left|b_{21}\right|\right) e^{\sigma \tau_{1}}+\left(\left|c_{11}\right|+\left|c_{21}\right|\right) e^{\sigma \varsigma_{1}}\right]=0.9249>0 \\
& d_{2}-\sigma-\frac{2}{3}\left[\left(\left|a_{12}\right|+\left|a_{22}\right|\right)+\left(\left|b_{12}\right|+\left|b_{22}\right|\right) e^{\sigma \tau_{2}}+\left(\left|c_{12}\right|+\left|c_{22}\right|\right) e^{\sigma \varsigma_{2}}\right]=0.8499>0
\end{aligned}
$$

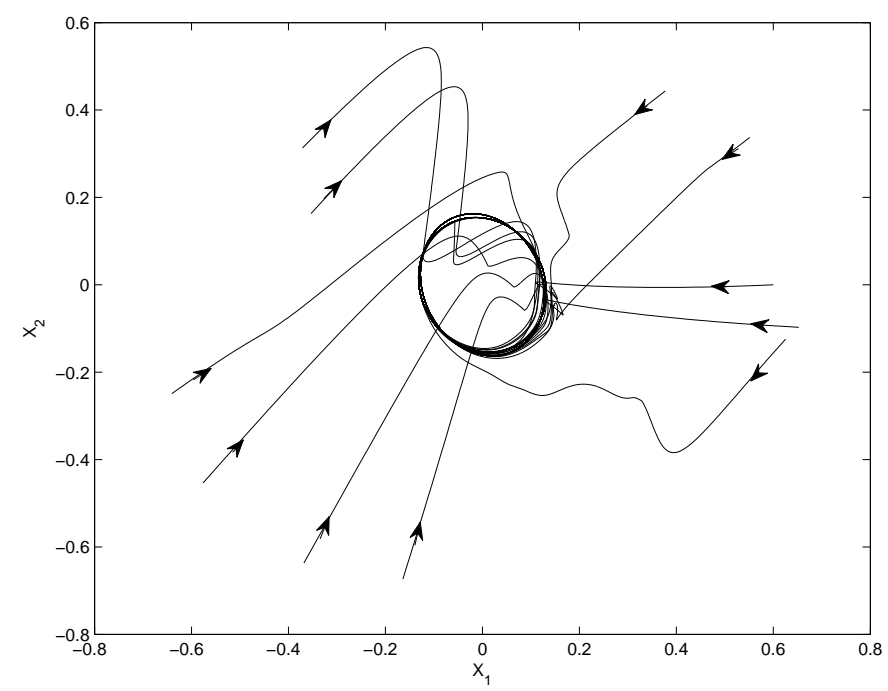

Fig. 1. Periodicity of RNNs (4.1) with proportional delays

Theorem 3.1 are satisfied. Thus, by Theorem 3.1, there exists one periodic solution of (4.1) with $2 \pi$ as its period and all other solutions of (4.1) converge exponentially to it as $t \rightarrow \infty$. The existence of exponentially periodic solutions can be clearly observed in Fig. 1.

Example 4.2 Consider the following system

$$
\dot{x}_{i}(t)=-d_{i} x_{i}(t)+\sum_{j=1}^{2}\left[a_{i j} f_{j}\left(x_{j}(t)\right)+b_{i j} g_{j}\left(x_{j}\left(p_{j} t\right)\right)+c_{i j} h_{j}\left(x_{j}\left(q_{j} t\right)\right)\right]+I_{i}(t),
$$

for $i=1,2, t \geq 1$. Where $d_{1}=4, d_{2}=3.5, a_{11}=1, a_{12}=0, a_{21}=-2, a_{22}=$ $1, b_{11}=-1, b_{12}=-2, b_{21}=0, b_{22}=1, c_{11}=0, c_{12}=1, c_{21}=1, c_{22}=0$. $I_{1}(t)=\sin (t), I_{2}(t)=\cos (t)$. The activation functions are $f_{i}\left(x_{i}\right)=\frac{1}{4}\left(\mid x_{i}+\right.$ $\left.1|-| x_{i}-1 \mid\right), g_{i}\left(x_{i}\right)=\cos \left(\frac{1}{2} x_{i}\right)+\frac{1}{4} x_{i}$ and $h_{i}\left(x_{i}\right)=\tanh \left(x_{i}\right)$ with Lipschitz constants $L_{i}=\frac{1}{2}, M_{i}=\frac{3}{4}$ and $N_{i}=1, i=1,2$. And $0<p_{j}, q_{j} \leq 1, j=1,2$.

We have

$$
\left\{\begin{array}{l}
d_{1}-\left[\frac{1}{2}\left(\left|a_{11}\right|+\left|a_{12}\right|\right)+\frac{3}{4}\left(\left|b_{11}\right|+\left|b_{12}\right|\right)+\left(\left|c_{11}\right|+\left|c_{12}\right|\right)\right]=0.25>0, \\
d_{2}-\left[\frac{1}{2}\left(\left|a_{21}\right|+\left|a_{22}\right|\right)+\frac{3}{4}\left(\left|b_{21}\right|+\left|b_{22}\right|\right)+\left(\left|c_{21}\right|+\left|c_{22}\right|\right)\right]=0.25>0 .
\end{array}\right.
$$




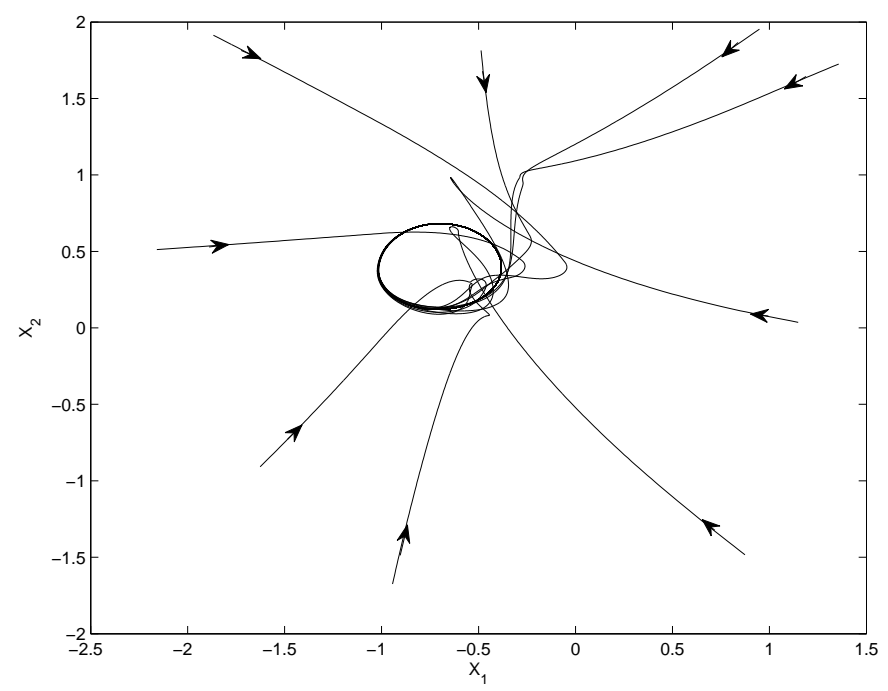

Fig. 2. Periodicity of RNNs (4.2) with proportional delays

The sufficient conditions in Theorem 3.2 are satisfied. Therefore, by Theorem 3.2, there exists one periodic solution of (4.2) with $2 \pi$ as its period and all other solutions of (4.2) converge exponentially to it as $t \rightarrow \infty$. The existence of exponentially periodic solutions can be clearly observed in Fig. 2 (Taking $\left.p_{j}=q_{j}=0.5, j=1,2\right)$.

Example 4.3 Consider the following system

$$
\dot{x}_{i}(t)=-d_{i} x_{i}(t)+\sum_{j=1}^{2}\left[a_{i j} f_{j}\left(x_{j}(t)\right)+b_{i j} g_{j}\left(x_{j}\left(p_{j} t\right)\right)+c_{i j} h_{j}\left(x_{j}\left(q_{j} t\right)\right)\right]+I_{i}(t),
$$

for $i=1,2, t \geq 1$. Where $d_{1}=3, d_{2}=4, a_{11}=0.5, a_{12}=1, a_{21}=1, a_{22}=$ $-1, b_{11}=0.5, b_{12}=-0.5, b_{21}=0.6, b_{22}=1, c_{11}=1, c_{12}=1, c_{21}=$ $0.5, c_{22}=1 . I_{1}(t)=\cos (2 t), I_{2}(t)=\sin (2 t)$. The activation functions are $f_{i}\left(x_{i}\right)=\tanh \left(\frac{1}{2} x_{i}\right), g_{i}\left(x_{i}\right)=\cos \left(\frac{1}{4} x_{i}\right)+\frac{1}{4} x_{i}$ and $h_{i}\left(x_{i}\right)=\frac{1}{4}\left(\left|x_{i}+1\right|-\left|x_{i}-1\right|\right), i=$ 1,$2 ;$ And $p_{j}=0.3, q_{j}=0.5, j=1,2$,

Obviously $f_{i}\left(x_{i}\right), g_{i}\left(x_{i}\right)$ and $h_{i}\left(x_{i}\right), i=1,2$ are Lipschitz continuous with Lipschitz constants $L_{i}=\frac{1}{2}, M_{i}=\frac{1}{2}$ and $N_{i}=\frac{1}{4}, \quad i=1,2 . \tau_{j}=-\log 0.3=$ $1.2040, \varsigma_{j}=-\log 0.5=0.6931, j=1,2$. Taking $\eta=0.5$, we have

$$
\begin{array}{r}
d_{1}-\eta-\left[\left(\left|a_{11}\right| L_{1}+\left|a_{12}\right| L_{2}\right)+\left(\left|b_{11}\right| M_{1} e^{\eta \tau_{1}}+\left|b_{12}\right| M_{2} e^{\eta \tau_{2}}\right)+\right. \\
\left.\left(\left|c_{11}\right| e^{\eta \varsigma_{1}}+\left|c_{12}\right| e^{\eta \varsigma_{2}}\right)\right]=0.1301>0 \\
d_{2}-\eta-\left[\left(\left|a_{21}\right| L_{1}+\left|a_{22}\right| L_{2}\right)+\left(\left|b_{21}\right| M_{1} e^{\eta \tau_{1}}+\left|b_{22}\right| M_{2} e^{\eta \tau_{2}}\right)+\right. \\
\left.\left(\left|c_{21}\right| e^{\eta \varsigma_{1}}+\left|c_{22}\right| e^{\eta \varsigma_{2}}\right)\right]=0.5091>0 .
\end{array}
$$

Theorem 3.3 are satisfied. Thus, by Theorem 3.3, there exists one periodic 


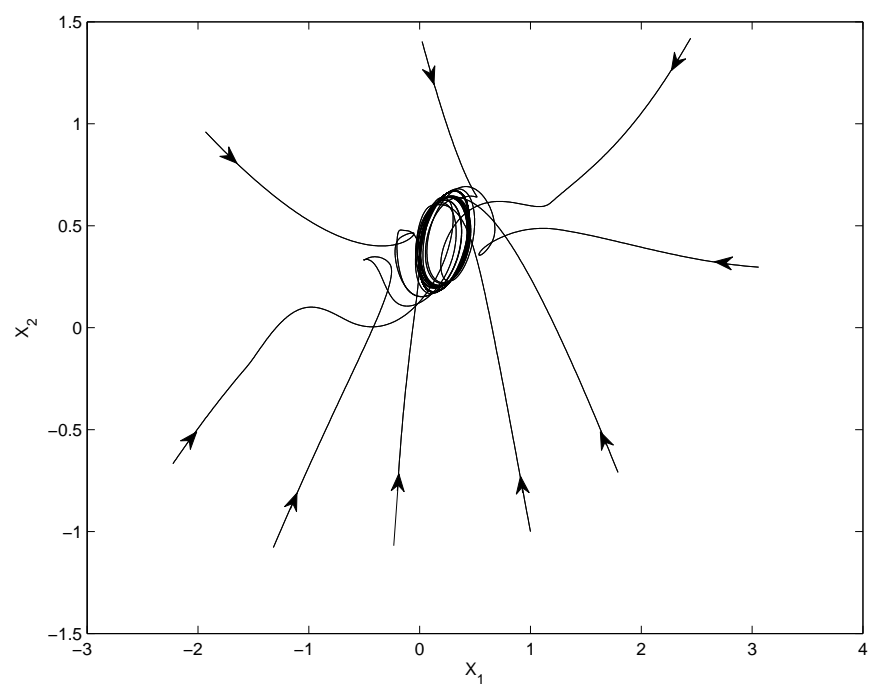

Fig. 3. Periodicity of RNNs (4.4) with proportional delays

solution of (4.4) with $\pi$ as its period and all other solutions of (4.4) converge exponentially to it as $t \rightarrow \infty$. The existence of exponentially periodic solutions can be clearly observed in Fig. 3.

Example 4.4 Consider the following system

$$
\dot{x}_{i}(t)=-d_{i} x_{i}(t)+\sum_{j=1}^{2}\left[a_{i j} f_{j}\left(x_{j}(t)\right)+b_{i j} g_{j}\left(x_{j}\left(p_{j} t\right)\right)+c_{i j} h_{j}\left(x_{j}\left(q_{j} t\right)\right)\right]+I_{i},
$$

for $i=1,2, t \geq 1$. Where $I_{1}=1, I_{2}=2$. The rest of this example are exactly the same as Example 4.2. So, (4.3) is also established.

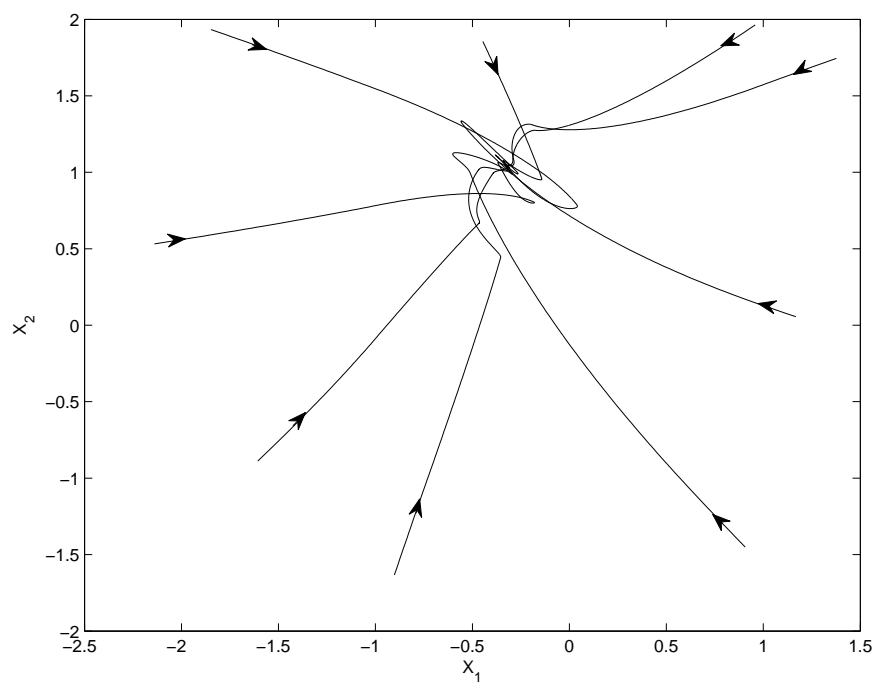

Fig. 4. Stability of RNNs (4.5) with proportional delays 
By Corollary 3.5, system (4.5) is globally exponentially stable, whose equilibrium point is $(-0.3318,1.0510)^{T}$ by using Matlab. The stability of system (4.5) can be clearly observed in Fig. 4.

\section{Conclusions}

In this paper, the exponential periodic and stability of recurrent neural networks with proportional delays have been studied. The activation functions only need to satisfy the Lipschitz conditions, which need not be differentiable, bounded and monotone increasing. The exponential stability criteria obtained are very simple and easily checked by simple algebraic methods. The nonlinear transform $y_{i}(t)=x_{i}\left(\mathrm{e}^{t}\right)$ can transform RNNs with proportional delays into RNNs with constant delays and time-varying coefficients. Therefore, the stability problem of neural networks with unbounded delay is changed into the stability problem of neural networks with constant time delays, so as to simplify the problem, which might have an impact role in studying the dissipativity, instability, and synchronization.

\section{Acknowledgments}

The author would like to thank the editor and the anonymous reviewer$\mathrm{s}$ for their valuable comments and constructive suggestions. The project is supported by the National Science Foundation of China (No.61374009).

\section{References}

[1] Cao J, Wang J. Global asymptotic and robust stability of recurrent neural networks with time delays. IEEE Transactions on Circuits and Systems-I 2005; $52(2): 417-426$.

[2] $\mathrm{Hu} \mathrm{L}$, Gao H, Zheng W. Novel stability of cellular neural networks with intervaltime varying delay. Neural networks 2008; 21(10):1458-1463.

[3] Huang C, Cao J. Almost sure exponential stability of stochastic cellular neural networks with unbounded distributed delays. Neurcomputing 2009;72(1315):3352-3356.

[4] Feng Z, Lam J. Stability and dissipativity analysis of distributed delay cellular neural networks. IEEE Transactions on Neural networks 2011; 22 (6):981-997.

[5] $\mathrm{Hu}$ J, Wang J. Global stability of complex-valued recurrent neural networks with time-delays. IEEE Transactions on Neural networks and Learning Systems $2012 ; 23(6): 853-865$. 
[6] Sun J, Chen J. Stability analysis of static recurrent neural networks with interval time-varying delay. Applied Mathematics and Computation 2013; 221(15):111-120.

[7] Liu P.L. Delay-dependent global exponential robust stability for delayed cellular neural networks with time-varying delay. ISA Transactions 2013;52:711-716.

[8] Chen B, Wang J. Global exponential periodicity of a class of recurrent neural networks with oscillating parameters and time-varying delays. IEEE Transactions on Neural networks 2005;16(6):1440-1448.

[9] Li C, Liao X. Robust stability and robust periodicity of delayed recurrent neural networks with noise disturbance. IEEE Transactions on Circuits and Systems-I 2006;53(10):2265-2273.

[10] Li Y, Zhu L, Liu P. Existence and stability of periodic solutions of delayed cellular neural networks. Nonlinear Analysis-Real World Applications 2006; $7(2): 225-234$.

[11] Yang Y, Cao J. Stability and periodicity in delayed cellular neural networks with impulsive effects. Nonlinear Analysis-Real World Applications 2007;8(1):362374 .

[12] Lou X, Cui B. Delay-dependent criteria for global robust periodicity of uncertain switched recurrent neural networks with time-varying delay. IEEE Transactions on Neural networks 2008;19(4):549-557.

[13] Huang Z, Xia Y. Exponential periodic attractor of impulsive BAM networks with finite distributed delays. Chaos, Solitons and Fractals 2009;39 (1):373-384.

[14] Tan M, Tan Y. Global exponential stability of periodic solution of neural network with variable coefficients and time-varying delays. Applied Mathematical Modelling 2009;33(1):373-385.

[15] Huang X, Cao J, Ho DWC. Existence and attractivity of almost periodic solution for recurrent neural networks with unbounded delays and variable coefficients. Nonlinear Dynamics 2006;45(3-4):337-351.

[16] Liu Y, Wang Z, Liu X. Stability criteria for periodic neural networks with discrete and distributed delays. Nonlinear Dynamics 2007;49(1-2):93-103.

[17] Zhou L, Hu G. Global exponential periodicity and stability of cellular neural networks with variable and distributed delays. Applied Mathematics and Computation 2008;195(2):402-411.

[18] Wang X, Li S, Xu D. Globally exponential stability of periodic solutions for impulsive neutral-type neural networks with delays. Nonlinear Dynamics 2011;64(1-2):65-75.

[19] Liu Y, Huang Z, Chen L. Almost periodic solution of impulsive Hopfield neural networks with finite distributed delays. Neural Computing and Applications $2012 ; 21(5): 821-831$. 
[20] Yang G, Kao Y, Li W, Sun X. Exponential stability of impulsive stochastic fuzzy cellular neural networks with mixed delays and reaction-diffusion terms. Neural Computing and Applications 2013;23(3-4):1109-1121.

[21] Xu H, Wu R. Periodicity and exponential stability of discrete-time neural networks with variable coefficients and delays. Advances in Difference Equations (2013) DOI: 10.1186/1687-1847-2013-226.

[22] Xu C, Zhang Q. Existence and exponential stability of anti-periodic solutions for a high-order delayed Cohen-Grossberg neural networks with impulsive effects. Neural Processing Letters 2014;40(3):227-242.

[23] Shao Y, Zhang Q. Stability and periodicity for impulsive neural networks with delays. Advances in Difference Equations (2013) DOI: 10.1186/1687-1847-20133529 .

[24] Yang W. Periodic solution for fuzzy Cohen-Grossberg BAM neural networks with both time-varying and distributed delays and variable coefficients. Neural Processing Letters 2014;40(1):51-73.

[25] Chiu K, Pinto M, Jeng J.C. Existence and global convergence of periodic solutions in recurrent neural network models with a general piecewise alternately advanced and retarded argument. Acta Applicandae Mathematicae 2014;133(1):133-152.

[26] Duan L, Huang L, Guo Z. Stability and almost periodicity for delayed high-order Hopfield neural networks with discontinuous activations. Nonlinear Dynamics 2014;77(4):1469-1484.

[27] Chen Y, Qiao C. Proportional differentiation: a scalable QoS approach. IEEE communications magazine 2003;41(6):52-58.

[28] Wei J, Xu C, Zhou X, Li Q. A robust packet scheduling algorithm for proportional delay differentiation services. Computer Communications 2006;29(18):3679-3690.

[29] Liu Y.K. Asymptotic behavior of functional differential equations with proportional time delays. European Journal of Applied Mathematics 1996;7(1):11-30.

[30] Iserles A. The asymptotic behaviour of certain difference equations with proportional delay. Applied Mathematics and Computation 1994;28(1-3):141152.

[31] Iserles A, Liu Y. On neural functional-differential equationals with proportional delays. Journal of Mathematical Analysis and Applications 1997;207(1):73-95.

[32] Zhou L. Dissipativity of a class of cellular neural networks with proportional delays. Nonlinear Dynamics 2013;73(3):1895-1903.

[33] Zhou L. Delay-dependent exponential stability of cellular neural networks with multi-proportional delays. Neural Processing Letters 2013;38(3): 321-346. 
[34] Zhou L, Chen X, Yang Y. Asymptotic stability of cellular neural networks with multi-proportional delays. Applied Mathematics and Computation 2014;229(1):457-466.

[35] Zhou L. Global asymptotic stability of cellular neural networks with proportional delays. Nonlinear Dynamics 2014;77(1):41-47.

[36] Zhou L. Novel global exponential stability criteria for hybrid BAM neural networks with proportional delays. Neurocomputing 2015;161:99-106.

[37] Zheng C, Li N, Cao J. Matrix measure based stability criteria for high-order networks with proportional delay. Neurcomputing 2015;149:1149-1154.

[38] Hiena L.V, Son D.T. Finite-time stability of a class of non-autonomous neural networks with heterogeneous proportional delays. Applied Mathematics and Computation 2015;14:14-23. 